\section{Comunicación y educación interactiva en salud y su aplicación al control del paciente diabético}

\author{
Rosario García González¹, \\ Rolando Suárez Pérez ${ }^{1} y$ \\ Oscar Mateo-de-Acosta ${ }^{1}$
}

\section{RESUMEN}

En este artículo se analiza la necesidad de participación de los grupos destinatarios para hacer más efectiva la educación en salud. Partiendo del enfoque tradicional de la educación, se profundiza en las distintas opciones de los procesos de comunicación y educación en este campo. En el modelo de comunicación participatoria, donde se desempeña la doble función de emisor y receptor, se llega a la coproducción de los mensajes de educación en salud. En vista de la experiencia del Programa de Educación en Diabetes del Instituto Nacional de Endocrinología de Cuba, se presenta un modelo de educación en salud basado en una estrategia de comunicación interactiva. Su objetivo es desarrollar en el sujeto la capacidad para pensar y decidir acerca de su salud partiendo de la discusión en grupos, facilitada por el proveedor de salud. En este modelo, el propósito y el contenido educativo los formulan los mismos beneficiarios. El punto de partida de la discusión se centra en el sujeto, tomando en cuenta sus necesidades de salud reales y sentidas. Se revisan los resultados obtenidos con esta metodología y se concluye que el modelo abre nuevas posibilidades educativas que facilitan la toma de decisiones en materia de salud y estilos de vida saludables.

\footnotetext{
Instituto Nacional de Endocrinología, La Habana, Cuba. Dirección postal: Instituto Nacional de Endocrinología, Hospital Cmdte. Manuel Fajardo Zapata y D. Vedado, La Habana 10400, Cuba.
}

En las ciencias médicas se utilizan con creciente frecuencia los programas de educación en salud en la comunidad (1). En ese contexto, la educación y la comunicación facultan a las personas a tomar decisiones en materia de salud y a seguir el tratamiento contra alguna enfermedad en particular para evitar su avance $(2,3)$. En este estudio se analizan ambos procesos teniendo en cuenta la experiencia en la educación de pacientes diabéticos y se propone una opción de educación interactiva en salud.

Lamentablemente, la educación en salud se ha visto limitada por la tendencia a considerarla un resultado de la acción de las instituciones sanitarias. Sin embargo, la educación en salud no es solo un producto (4), sino también un proceso que es responsabilidad de los servicios de salud, las instituciones sociales y la población a la que está dirigida la intervención educativa. Por otra parte, la formación del personal de salud y la orientación tradicional de las estrategias de comunicación han tenido un carácter eminentemente biológico y "unidireccional" (5), en vez de incorporar estrategias participatorias.

El diseño de estrategias de comunicación en salud es una tarea compleja $(2,3)$. Cada problema sanitario tiene características muy particulares; tan solo mencionar algunas enfermedades despierta temor y provoca reacciones emocionales en el público. Una conducta terapéutica prescrita puede ser ajena o incluso contraria a los hábitos de la población. En el caso de las enfermedades crónicas, existe cierto grado de frustración del paciente ante la incapacidad del médico para resolver el problema de manera definitiva.

Para controlar esos efectos negativos hay que tener en cuenta la opinión de la población sobre sus necesidades de salud reales y sentidas, que generalmente es distinta a la del proveedor de los servicios sanitarios. También hay que conocer y respetar los valores, tradiciones y estereotipos de las poblaciones. Finalmente, se debe fomentar la responsabilidad individual y comunitaria por medio de métodos participatorios de educación y comunicación. Hasta ahora estos se han basado en lo que indican las "prácticas de la buena salud" y en "lo que debe hacerse" pero no en las necesidades de la población destinataria.

La comunicación y la educación están estrechamente ligadas (6). Ambas comparten objetivos comunes y están conectadas con las características esenciales del ser humano de comunicarse y apren- 
der. No obstante, son independientes. Por medio del aprendizaje, el ser humano adquiere conocimientos, habilidades y actitudes y desarrolla diferentes formas y grados de recibir y responder a los mensajes de salud. Por otra parte, la capacidad de comunicación entre emisor y receptor determina el grado de aprovechamiento del mensaje.

$\mathrm{Al}$ analizar el proceso de enseñanza y aprendizaje se encuentra que la forma tradicional de enseñanza se ha basado en un esquema de comunicación lineal donde el educador emite un estímulo para provocar una respuesta en el receptor (7). A pesar de ser fuertemente criticado por las corrientes pedagógicas modernas, que promueven una mayor participación del educando, ese paradigma ha sido el más utilizado en campañas de educación en salud.

En contraposición han surgido modelos educativos basados en el diálogo, que establecen una comunicación horizontal o igualitaria y buscan más retroalimentación o participación (8). Esos sistemas educativos se han incorporado a la comunicación social en salud de acuerdo con los principios de la medicina comunitaria aceptados universalmente a partir de la Declaración de Alma-Ata. Esta tendencia, que en América Latina tomó auge en la década de 1960 mediante las teorías críticas latinoamericanas $(9,10)$, se manifiesta en los modelos de comunicación en grupos y comunicación participatoria (11). El primer modelo implica dos fases de comunicación: primero la audición o visión del mensaje y, posteriormente, la reflexión y discusión. Por otra parte, en la comunicación participatoria cada miembro del grupo desempeña las funciones de emisor y receptor para convertirse en coautor de los mensajes. Esos modelos se caracterizan por hacer más hincapié en los conceptos, necesidades, contenidos y métodos que en los medios. Los beneficiarios establecen los objetivos de acuerdo con sus necesidades, pero orientados por el facilitador. Como ejemplo de la aplicación de estos principios, a continuación se presenta un modelo de educación en salud basado en una estrategia de comunicación interactiva, preparada por el Programa de Educación en Diabetes del Instituto Nacional de Endocrinología de Cuba.

\section{MODELO DE EDUCACIÓN EN SALUD PARA EL PACIENTE DIABÉTICO}

Muchos autores reconocen que la educación es un componente vital de la atención del paciente diabético $(5,12,13)$. Sin embargo, la mayor parte de las estrategias educativas más comunes se basan en consejos autocráticos que responden al modelo tradicional de comunicación. Estas se oponen a la formación de un individuo consciente de su problema de salud y responsable de su cuidado diario.
De acuerdo con los elementos de la comunicación establecidos por Prieto (14), la educación del paciente diabético presenta las siguientes características: generalmente, el personal de salud desempeña la función del emisor; en ocasiones, estas personas utilizan términos científicos que muchos pacientes no comprenden; en cuanto al mensaje, su formulación descansa exclusivamente en el emisor. Se abusa de los términos "debe", "tiene" y "es mejor que".

Asimismo, los mensajes que predominan están destinados a enseñar al grupo destinatario a promover su propia salud. Son menos frecuentes aquellos que procuran determinar las necesidades reconocidas por el grupo y los mensajes provenientes del propio grupo destinatario, que facilitan el intercambio de opiniones, sentimientos y experiencias, así como la producción de nuevos mensajes. En cuanto al marco de referencia, predomina la relación entre el paciente y la enfermedad cuando el contenido del mensaje se centra en las exigencias terapéuticas más que en las necesidades individuales de cada paciente. El receptor es pasivo y no tiene grandes posibilidades de emitir su opinión.

Si lo que se busca al educar al paciente diabético es capacitarlo para que ajuste su estilo de vida y tome decisiones ante las exigencias diarias del tratamiento, el modelo pedagógico basado en el asesoramiento directo contradice el objetivo planteado. Los resultados de las diferentes evaluaciones sobre la educación de diabéticos realizadas en el Instituto Nacional de Endocrinología de Cuba (15) aportaron información que permitió elaborar una estrategia de comunicación interactiva que facilita la formación de un individuo responsable por su estado de salud $y$, por lo tanto, capaz de modificar su conducta.

El modelo pedagógico busca desarrollar en el paciente su capacidad para pensar y decidir, junto con el médico, cuáles son sus necesidades de salud. Este modelo se basa en la discusión en grupos y en la relación interpersonal. Usa un lenguaje de fácil comprensión para el paciente y diseña situaciones de aprendizaje en que este puede participar activamente. Se vale de la aplicación de diferentes técnicas participatorias, como el modelo interactivo (16) y el metaplán (17), así como de diversas técnicas de animación, análisis y reflexión (18).

En este modelo rige una comunicación participatoria $(11,19)$ en la que cada miembro del grupo cumple con las funciones de emisor y receptor para coproducir un mensaje a partir de una información de base. Este principio se mantiene en la relación interpersonal del proveedor de salud con cada uno de los pacientes.

La experiencia del grupo se fundamenta en el trabajo en conjunto que facilita el desarrollo de la actividad cognoscitiva, la cooperación, y la 
búsqueda de diferentes soluciones. Posteriormente, esas acciones se refuerzan en el contacto individual entre el proveedor de salud y el paciente.

Los objetivos y el contenido los establecen los mismos pacientes. El centro de la discusión no es la diabetes, sino el individuo y su salud en relación con su entorno y con sus necesidades, expresadas en un ambiente informal y espontáneo que facilita la comunicación de los sentimientos con seguridad y libertad.

A continuación se describe el modelo educativo:

- Mensaje. Se apoya en un lenguaje sencillo y práctico, aborda mensajes "para", "sobre" y "de" los diabéticos y es coproducido en la discusión de grupo.

- Emisor y receptor. Todos los participantes desempeñan ambas funciones, lo que rompe el esquema unidireccional de comunicación para establecer la interacción en un diálogo abierto y espontáneo. Hay que superar el concepto tradicional del consejero autocrático que indica al grupo "lo que debe" y "lo que no debe" hacer y actuar como el promotor de la discusión y de la búsqueda de opciones de solución. En una participación responsable, se comparten las opiniones y experiencias para lograr el enriquecimiento del mensaje final.

En el caso de los familiares del paciente, les corresponde una triple función. En primer lugar, son sujeto y objeto de educación, pues el familiar es un individuo que requiere de información y un mínimo de conocimientos para convivir con un paciente diabético y ayudarle a sobrellevar las exigencias terapéuticas. Como apoyo del paciente en el hogar y en la comunicación con los servicios de salud, el familiar contribuirá a que el diabético siga el tratamiento pero sin sobreprotegerlo ni asumir la responsabilidad que incumbe al paciente por su estado de salud. Finalmente, se desempeña en la familia y en la comunidad como promotor de hábitos y estilos de vida sanos mediante su participación en las actividades para diabéticos y de fomento de la salud.

- Código o lenguaje. Ha de ser simple y fácil de comprender, independientemente del nivel de escolaridad y grupo de edad de cada paciente.

- Medios y recursos. No se requiere de medios y recursos tecnológicos, aunque pueden incorporarse de acuerdo con los objetivos propuestos y las posibilidades. Hay que tener en cuenta que su único fin es facilitar la comunicación.

- Referente. El tema del mensaje no tratará sobre la diabetes sino sobre el paciente diabético, puesto que es a él a quien se quiere prestar la atención de salud.
- Marco de referencia. Durante la discusión, reflexión y toma de decisiones en materia de salud se deberán tener en cuenta el entorno familiar y la función social que desempeñan los pacientes.

- Formación social. Se relaciona directamente con las prioridades y políticas que se establezcan para preservar la salud de la población y para garantizar una buena atención médica y servicios de calidad.

\section{APLICACIÓN PRÁCTICA DEL MODELO}

De 1989 a 1994, en el Centro de Atención al Diabético (CAD) del Instituto Nacional de Endocrinología (INEN) se realizó un estudio prospectivo, antes y después de la intervención, en que se siguió a un grupo de 148 pacientes. Se incluyó a todos los pacientes con diabetes no insulinodependiente de 60 o más años de edad que consultaron al Centro y que aceptaron participar en un grupo educativo que celebrara reuniones mensuales. Se excluyó a los pacientes que presentaban complicaciones graves y a los que tenían discapacidad mental, trastornos psiquiátricos $\mathrm{u}$ otros problemas del habla o de la audición que afectaran su capacidad para comunicarse $(15,20,21)$. En el cuadro 1 se muestran los resultados: mayor cumplimiento del tratamiento, disminución del uso de insulina e hipoglucemiantes orales, y reducción del sobrepeso, así como disminución de los ingresos hospitalarios y mejoramiento del control metabólico.

Por otra parte, en un estudio de casos y controles (22) llevado a cabo de 1992 a 1993 en el Centro de Atención al Diabético del INEN, se siguió durante un año a un grupo de diabéticos insulinodependientes. Se seleccionaron los primeros 80 pacientes que acudieron a consulta en el período de reclutamiento. El tamaño de la muestra se determinó por procedimiento estadístico y tomando como variable de referencia el control metabólico según las concentraciones de hemoglobina glucosilada.

Se tomaron como criterios de exclusión al seleccionar la muestra el no haber terminado la enseñanza primaria, el haber recibido anteriormente educación sobre diabetes y el haber tenido complicaciones graves como consecuencia de la enfermedad, tales como ceguera o amputación de miembros. Se compararon los conocimientos sobre diabetes y cumplimiento con el tratamiento entre pacientes educados por el método de orientación tradicional $(n=30)$ e individuos que siguieron el método de educación interactiva $(n=50)$. Los resultados, que se resumen en el cuadro 2, mostraron que los conocimientos y destrezas de los pacientes incorporados al modelo educativo interactivo aumentaron significativamente en relación con los de pacientes seguidos 
CUADRO 1. Impacto de la educación interactiva en diabéticos no insulinodependientes mayores de 60 años $(n=148)$

\begin{tabular}{|c|c|c|c|}
\hline Variable & Al inicio & $\begin{array}{l}5 \text { años } \\
\text { después }\end{array}$ & $P$ \\
\hline $\begin{array}{l}\text { Pacientes que siguen } \\
\text { la dieta }\end{array}$ & $35,1 \%$ & $83,1 \%$ & $<0,001$ \\
\hline $\begin{array}{l}\text { Pacientes que siguen el } \\
\text { autocontrol diario }\end{array}$ & $28,4 \%$ & $93,9 \%$ & $<0,001$ \\
\hline $\begin{array}{l}\text { Pacientes que practican } \\
\text { ejercicio físico }\end{array}$ & $15,5 \%$ & $86,2 \%$ & $<0,001$ \\
\hline $\begin{array}{l}\text { Pacientes con sobrepeso } \\
\text { u obesidad }\end{array}$ & $77,0 \%$ & $20,0 \%$ & $<0,001$ \\
\hline $\begin{array}{l}\text { Pacientes con signos } \\
\text { de depresión }\end{array}$ & $69,0 \%$ & $23,6 \%$ & $<0,001$ \\
\hline $\begin{array}{l}\text { Dosis media de } \\
\text { glibenclamida }\end{array}$ & 25 mg/día & 10 mg/día & $<0,02$ \\
\hline $\begin{array}{l}\text { Dosis media de insulina } \\
\text { lenta }\end{array}$ & 20 U/día & 10 U/día & $<0,02$ \\
\hline $\begin{array}{l}\text { Valores medios de } \\
\text { hemoglobina } A_{1 C} \\
\text { Valores medios de alucosa }\end{array}$ & $12 \%$ & $8 \%$ & $<0,02$ \\
\hline $\begin{array}{l}\text { en orina } \\
\text { Pacientes que utilizan }\end{array}$ & $15-20 \mathrm{~g} / \mathrm{dL}$ & $0-5 \mathrm{~g} / \mathrm{dL}$ & $<0,001$ \\
\hline servicios de urgencia & $96,6 \%$ & $1,3 \%$ & $<0,0001$ \\
\hline $\begin{array}{l}\text { Hospitalizaciones/ } \\
\text { paciente/año }\end{array}$ & $2-3$ & $<1$ & $<0,001$ \\
\hline
\end{tabular}

CUADRO 2. Impacto del proceso educativo en pacientes insulinodependientes un año después del inicio de la intervención $(n=80)$

\begin{tabular}{lccc}
\hline & \multicolumn{3}{c}{ Metodología educativa } \\
\cline { 2 - 4 } \multicolumn{1}{c}{ Variable } & Tradicional & Interactiva & $P$ \\
\hline $\begin{array}{l}\text { Promedio de calificación } \\
\text { en la prueba de } \\
\text { conocimientos }\end{array}$ & 85,7 & 93,8 & $<0,05$ \\
$\begin{array}{l}\text { Pacientes con } \\
\text { conocimientos sobre } \\
\text { requerimientos alimentarios }\end{array}$ & $30,0 \%$ & $70,0 \%$ & $<0,02$ \\
$\begin{array}{l}\text { Pacientes con conocimientos } \\
\text { sobre hipoglicemia }\end{array}$ & $20,0 \%$ & $74,0 \%$ & $<0,02$ \\
$\begin{array}{l}\text { Pacientes que siguen la dieta } \\
\begin{array}{l}\text { Pacientes que siguen el } \\
\text { autocontrol diario }\end{array}\end{array}$ & $6,7 \%$ & $84,0 \%$ & $<0,02$ \\
$\begin{array}{l}\text { Valores medios de } \\
\text { hemoglobina } \mathrm{A}_{1 \mathrm{C}}\end{array}$ & $35,3 \%$ & $76,0 \%$ & $<0,02$ \\
\hline
\end{tabular}

con la orientación tradicional $(P<0,05)$. La adhesión al tratamiento fue significativamente mayor $(P<$ $0,02)$, así como el incremento de sentimientos de responsabilidad, autonomía y seguridad ante el tratamiento $(P<0,01)$. Esos resultados se reflejaron en una disminución significativa de los niveles de glucosa en sangre $(P<0,02)$. La estrategia también resultó eficaz en la detección de necesidades educativas en las áreas de atención primaria ${ }^{2}$ y en la capacitación de los proveedores de salud (23), habiéndose encontrado un año después de los talleres metodológicos diferencias significativas $(P<0,05)$ en los cuidados prácticos del paciente diabético y un aumento de la interacción disciplinaria $(P<0,01)$.

\section{CONCLUSIONES}

Tradicionalmente, en los servicios sanitarios se ha considerado al proveedor de salud como la única autoridad en la materia. Su formación solía responder a un enfoque eminentemente biológico y el trabajo interdisciplinario era mínimo.

A partir de Alma-Ata, el concepto de la salud comunitaria impuso un enfoque mucho más amplio en el que se han integrado un análisis multifactorial de los problemas de salud y una acción interdisciplinaria para solucionarlos. Este nuevo concepto hace hincapié en la comunicación y educación en salud por medio de la participación comunitaria. Además, paulatinamente van ocupando un lugar importante los modelos de educación horizontales y participatorios.

Como en el caso de los pacientes diabéticos en los países de América Latina, a pesar de que hay consenso sobre la necesidad de su participación activa y responsable en su propio cuidado y control diario, generalmente los programas establecidos no utilizan modelos de comunicación que promuevan esa participación. El modelo de educación interactiva anteriormente presentado abre nuevas vías de expresión e interacción que facilitan la coproducción de mensajes de acuerdo con el entorno social de los pacientes diabéticos. La reflexión y el análisis de las situaciones cotidianas, la identificación de las necesidades de salud y la toma de decisiones en conjunto enriquecen las experiencias de todos los que participan en el programa.

Estas estrategias educativas redundarán en mayor eficacia en el logro de los objetivos de la educación para la salud. Pueden ser igualmente útiles en cualquier programa para el control de enfermedades crónicas no transmisibles o en cualquier intervención para promover estilos de vida saludables en distintos grupos de población.

2 García R, Suárez R. “Diagnóstico participativo para la educación en diabetes en la atención primaria de salud." Ponencia presentada en el III Seminario Internacional de Atención Primaria de Salud. La Habana, Cuba, 1993. 


\section{REFERENCIAS}

1. Organización Mundial de la Salud. Nuevos métodos de educación sanitaria en la atención primaria de salud. Ginebra: OMS; 1983. (Serie de informes técnicos 690).

2. Organización Panamericana de la Salud. Manual de comunicación social para programas de salud. Washington, DC: OPS; 1992.

3. Organización Mundial de la Salud. Comunicación, planificación, administración y evaluación de las actividades de comunicación. Ginebra: OMS; 1987.

4. Franco S. Orientación biosocial de la educación y salud en la sociedad. Educ Med Salud 1990;24:103-114.

5. Assal J. A global integrated approach to diabetes: a challenge for more efficient therapy. En: Davidson JK, ed. Clinical diabetes mellitus: a problem oriented approach. New York: Thieme Stratton; 1985.

6. Orozco G. Comunicación y modelos educativos. Módulo III: comunicación educativa y cultural. México, DF: Instituto Latinoamericano de Comunicación Educativa; 1991.

7. Berlo DK. El proceso de la comunicación: introducción a la teoría y a la práctica. En: De Anda ML. Introducción a la tecnología educativa: módulo propedéutico. México, DF: Instituto Latinoamericano de Comunicación Educativa; 1991.

8. Goffman E. Ritual de la interacción. Buenos Aires: Tiempo Contemporáneo; 1970.

9. Atwood R, McAnany E, eds. Communication and Latin American Society. Madi- son: University of Wisconsin Press; 1986.

10. Instituto Latinoamericano de Comunicación Educativa, Organización de los Estados Americanos. Maestría en tecnología educativa: las teorías críticas latinoamericanas. México, DF: ILCE-OEA; 1991.

11. Kaplun M. Hacia nuevas estrategias de comunicación en la educación de adultos. Santiago, Chile: Organización de las Naciones Unidas para la Educación, la Ciencia y la Cultura; 1983.

12. Groen JJ, Pelser HE. Newer concepts of teaching, learning and education and their application to the patient-doctor cooperation in the treatment of diabetes mellitus. Pediatr Adolesc Endocrinol 1992;10:168-177.

13. Assal J, Conti A. Education: still the most important basis for treatment. Int Diabetes Found Bull 1988;33:55-60.

14. Prieto D. Comunicación y percepción en las migraciones. Barcelona: Serbal/UNESCO; 1984.

15. García R, Suárez R. La educación al paciente diabético. La Habana: Editorial Ciencias Médicas; 1992.

16. Carlson A, Rosenqvist U. Locally developed plan for quality diabetes care: worker and consumer participation in the public health-care system. Health Educ Res 1990;5:41-52.

17. Schnelle W. International training and learning: the metaplan method. En: Assal J PH, Berger M, Gay N, Canivet J, Diabetes Education Study Group
(EASD), eds. Diabetes education: how to improve patient education. Excerpta Medica 1983;624-628.

18. Vargas L. Técnicas participativas para la educación popular. San José, Costa Rica: Alforja; 1984

19. Prieto D. Discurso autoritario y comunicación alternativa. México, DF: Premiá; 1986.

20. García R, Suárez R. Diabetes education in the elderly: a 5-year follow-up of an interactive approach. Patient Educ Couns 1996:29:87-97.

21. García R, Suárez R, Mateo de Acosta O. Programa de educación en diabetes del Instituto Nacional de Endocrinología en Cuba: una estrategia de comunicación interactiva. Rev Asoc Latinoamer Diabetes 1993;3:112-115.

22. García R, Suárez R, Gómez J, Romero MI, Portilla L. Educación interactiva vs. educación convencional en diabéticos insulino dependientes. Av Diabetologia 1994;9:45-62.

23. Suárez R, García R. Educating diabetes educators: an interactive methodology and an interdisciplinary team. Patient Educ Couns 1994;23(suppl 1):S78.

Manuscrito aprobado para publicación en versión revisada el 21 de mayo de 1996.
ABSTRACT

Health communication and interactive education and their application to control of the diabetic patient
This article analyzes the need for participation of the groups that are the recipients of health messages in order to make health education more effective. Taking the traditional educational approach as the point of departure, the different options for communication and education processes in this field are explored in depth. The participatory communication model, in which the subject plays the role of both transmitter and recipient, leads to the coproduction of health education messages. In light of the experience of the Diabetes Education Program of the National Endocrinology Institute of Cuba, a health education model based on a strategy of interactive communication is presented. Its objective is to develop in the patient the capability to think and decide about his or her health through discussion groups facilitated by a health care provider. In this model, the beneficiaries of the health message help shape its aim and content. The discussion centers on the patient, taking into account his or her real health needs and feelings. The results obtained with this methodology are reviewed, and it is concluded that this model opens new educational possibilities that will facilitate decision-making with regard to health and healthy lifestyles. 